\title{
Identidades en movimiento: la danza y la experiencia formativa en cinco bailarinas-estudiantes universitarias
}

\author{
Identities in movement: dance and formative experience in five \\ dancers-students university
}

ADRIANA BUSTOS* Y RODRIGO DÍAZ**

\begin{abstract}
Resumen: Se revisa la problemática general de la identidad y el cuerpo desde perspectivas biológicas, antropológicas y filosóficas, con especial énfasis en el desarrollo de la antropología del cuerpo y de la teoría del sujeto en dos vertientes: Judith Butler y el último Michel Foucault. En ese sentido se establece la danza y su relación con el cuerpo como un espacio/objeto de interés. Se exponen sucintamente los paradigmas de la neuro-estética y la neurobiología y se explicita su modelo epistémico. Se especifica de qué manera esa episteme se relaciona con las teorías del sujeto y cómo las transforma. Se señala su carácter contingente e histórico. Por último se presentan los resultados de un breve trabajo etnológico que señala la importancia que tienen las ciencias humanas para mediar con el paradigma de las neurociencias y evitar determinismos.
\end{abstract}

\begin{abstract}
Article is look on the general problematic of body/identity from different perspectives: biological, anthropological and philosophical, with especial emphasis on de development of the anthropology of the body and subject theory: Both Judith Butler and the last Michel Foucault. Dance is used as an object of research. A succinct review on the neuro-aesthetic approach to dance will help reveal the neuro sciences as an emerging epistemic system which has interesting implications to subject theory. Lastly those theories are compared with a short ethnological work that uses the life stories of 4 non-professional dancers and delves into the role dance has had on their life and how does it relates to their personal and professional life.
\end{abstract}

There is a certain departure from the human that takes place in order to start the process of remaking the human. Judith Butler Undoing Gender pág 4.

\section{Problemática: identidad y cuerpo}

El cuerpo constituye los límites de la identidad, el principio de la acción pública y la arena sobre la que interactuamos con los demás. Desde una perspectiva somos agentes cor-

Fecha de recepción: 10/06/2016. Fecha de aceptación: 20/07/2016.

* Universidad de Salamanca.

** Estudiante de doctorado. Universidad de Salamanca. Contacto: diaz.bocanegra@gmail.com 
póreos encarnados (Esteban 2001) con necesidades materiales, deseos y aspiraciones. Estos agentes corpóreos son a su vez una manifestación del individuo biológico como sujetos que se constituyen sobre y desde el cuerpo y sobre los que se ejerce un reconocimiento a través de categorías que pueden llegar a ser inmobilizantes o violentas, porque no reconocen el devenir inherente a las historias de vida (Butler 2004; 80).

La violencia ética una vez internalizada limita al sujeto en sus posibilidades de ser. Le asigna funciones adecuadas y define el rango de las estrategias posibles del sujeto dentro de la arena pública. Sobre el cuerpo impone el género y las expectativas sociales de ese cuerpo-género. La identidad queda trastocada, atada en unas categorías fijas sujetadas no desde el exterior sino desde el propio sujeto por mecanismo psíquicos y éticos (Butler 2010, Thielm 2012). Esa sujeción puede causar un desasosiego y angustia al no corresponderse con la realidad ni las preguntas que encuentra el sujeto en su historia de vida.

Así, en vez de un continente biológico, el cuerpo queda enfrentado con el sujeto. Se transforma en una jaula de la identidad por los atributos que se le adhieren y las expectativas que se le imponen. Pero ¿Qué queda de ese cuerpo humano sin todos esos atributos? ¿Qué posibilidades se tienen para de construirse y abrirse a nuevas posibilidades de la identidad?

El trabajo que presentamos parte de estas preguntas para cuestionar a la danza como un doble espacio, por un lado el de un espacio en el que se aprecia el cruce entre las epistemes contemporáneas de la biología, la neurología y la antropología del cuerpo; por otro un espacio de práctica "dietética" que funciona como una hermenéutica del sujeto para los practicantes de la disciplina, sujetos que encarnan esas epistemes y las habitan.

\section{2. ¿Pero, por qué la danza?}

El cuerpo en su complejidad es el espacio de contienda de las identidades y categorías del pensamiento en su oposición dogmática tradicional objeto/sujeto, cuerpo/mente femenino/masculino, y el arte ha tenido históricamente una relación fundamental con el cuerpo, sea para admirarlo y reproducirlo, creando ideales, o bien para conformarlo a través de las artes expresivas como la danza. La cual está inmersa en otro eje del uso del cuerpo, el de la dietética entendida ampliamente como la alimentación y el deporte.

La danza, con el nivel de exigencia que plantea a sus practicantes, resulta especialmente violenta en términos identitarios (no siempre con efectos negativos) puesto que impone al cuerpo una transformación y una adecuación a un fin concreto e impone al sujeto una identificación tan fuerte con ese uso del cuerpo que el sujeto termina siendo un cuerpo. Asimismo, la danza es una actividad que se inicia en una edad muy temprana y que tiene un sesgo de género muy marcado. Con la correspondiente carga identitaria y estética. Cobra una importancia capital el estilo y el propósito de la formación en danza como mediadora de estos procesos. Asimismo, la danza es una actividad que se inicia en una edad muy temprana y que tiene un sesgo de género muy marcado. Con la correspondiente carga identitaria y estética.

Sin embargo, la danza también ofrece un espacio de liberación. Un momento de extrañamiento en el que sólo se es un cuerpo en movimiento potencialmente libre de atributos y categorías. Presenta además dos componentes fundamentales para la apertura: la emotividad 
y la empatía (Iacoboni 2009, Rizzolatti \& Craighreo 2004). El movimiento en su relación con la música, deja de ser únicamente una forma de deporte para comunicarse con las áreas límbicas del cerebro.

Veamos algunos de los paradigmas más importantes.

\section{Nuevos paradigmas sobre la relación danza-cuerpo-identidad}

Para entendernos, nosotros orientamos este trabajo desde la reflexión que Louis Waquant hizo sobre la disciplina corporal en el boxeo:

Una intensiva manipulación del organismo, regulada con precisión cuyo objetivo es introducir en el sistema corporal del púgil, series de rutinas de movimiento y estados subjetivos emocional-cognitivos que le convierten en un practicante dialogante de la dulce ciencia de golpear. Es una forma de trabajo práctico en el sentido que implica el ejercicio de una inteligencia que se realiza en comunicación con las realidades concretas y actuales de su entorno natural y su objeto (Bittner 1983, p. 253) Y reorganiza de forma práctica el campo corporal entero del púgil haciendo resaltar ciertos órganos y capacidades, y haciendo retroceder otros, transformando no sólo el físico del boxeador sino también el sentido de su cuerpo, la conciencia que tiene de su propio organismo y, a través de su cuerpo transformado, del mundo a su alrededor (Wacquant, 1995, citado en Esteban 2004)1․

La danza, creemos, tiene estos elementos de manipulación del organismo en función de objetivos en los que la toma de conciencia de la transformación corporal toma un lugar central. Ahora bien, dentro de esta transformación, la bailarina experimenta el mundo como un acto alrededor de su cuerpo-conciencia, lo que implica una reinterpretación -consciente o no- del entendimiento humano que en occidente no hace participe al cuerpo del conocimiento del mundo ni de la interpretación de la realidad, ni del acto comunicativo, al menos no en igualdad de jerarquía respecto a la mente.

Haremos ahora un breve resumen de los más recientes debates en neurobiología, en especial el interesante campo de la Neuroestética porque utiliza cada vez más a la danza como un referente.

\subsection{Neuroestética}

You ask me what is dance, and in school I learned it is time space and intensity, and I think that really is all that what dance is" Trisha Brown

Beatriz Calvo Merino, una de las autoridades más reconocidas en este campo habla del avance significativo en las técnicas de resonancia magnética (FMRI) que permiten estudiar los mecanismos neuronales involucrados en la experiencia estética de los trabajos

1 Esteban (2001) toma el concepto de Wacquant y lo relaciona con Capital en el sentido de bordieu, tal que si un boxeador invierte en su cuerpo, capitaliza ese trabajo como un rendimiento. 
de arte (Christensen, J.F. \& Calvo-Merino, B., 2013) la danza en este paradigma estaría caracterizada por tres elementos clave: Cuerpo, Movimiento, Emoción.

Observando estos estímulos a nivel cerebral, se teoriza que hay algo más que un tipo de patrón motór del cuerpo (Christensen, JF, \& Calvo-Merino, 2013). En concreto una región del cortex extrastriado, la EBA por su siglas en inglés o el área corporal extraestriada, responde a la visión de los cuerpos humanos (Downing, Jiang, Shuman \& Kanwisher, 2001). Se observa sin embargo, una complejidad en el procesamiento neuronal del movimiento y el cuerpo, donde se solapan apreciación y movimiento, al punto en que parece ser que "el cerebro es capaz de extraer el sentido de movimiento incluso de una representación estática del mismo" (Christensen \&Calvo-Merino, 2013; 5).

Así, dada la complejidad que involucra crear y ejecutar un movimiento de danza y dada la complejidad neuronal de su apreciación, la cuestión central de la neuro-estética es la transformación de la experiencia de la bailarina a la de la espectadora. Este paradigma redirige a la investigación seminal del grupo de Parma sobre las neuronas espejo. (Rizzolatti 1996 y 2011) para hablar entonces de una "resonancia" en el sistema neuro-motor del espectador al observar la danza que se transforma en una experiencia estética.

¿Cómo? Se teoriza que las respuestas simétricas observadas en distintas áreas del cerebro y el sistema nervioso muestran una comunión de la experiencia emotiva ${ }^{2}$ gracias a una: "especialización del sistema cerebral que permite a las personas percibir y procesar emociones transmitidas por expresiones faciales y otras claves de movimiento con contenido emocional (Christensen \& Calvo-Merino 2013, Blassing et al 2012)" Un área del cerebro que parece ser importante es el cortéx ventral premotor puesto que codifica los estímulos relacionados con la acción. Y que sería crucial en procesos coreográficos en los que los bailarines deben participar de los movimientos de los demás para coordinar y armonizar (Calvo-Merino et al 2010).

Ahora bien, dentro de estas interpretaciones, existen dos tipos de preguntas una pregunta suave, que se expresa en términos puramente técnicos. Y una pregunta fuerte que subyace y evita la simplificación.

- Pregunta suave: ¿Cómo se transforma un impulso eléctrico entre sinapsis en una forma compleja de movimiento?

- Pregunta dura: ¿Cómo estas sinapsis se transforman en sentimientos, en experiencia subjetiva, en experiencia objetiva?

Estas dos preguntas nos permiten diferenciar el nivel puramente biológico de la neurociencia y el nivel que intenta dar razón de un proceso más complejo, que constituye en última instancia el objeto de estudio de la neuro-estética y que tiene ramificaciones para la filosofía, la estética y la antropología. Implica un horizonte epistémico sobre la apreciación, la experiencia y la percepción.

2 Actividad en el suculo temporal superior, en el precuneo y el nucleo genicular. En la ínsula y el cortex cingular anterior (Petrini, Crabbe, Sheridan \& Pollick 2011) 
Ejemplos más consistentes respecto a la respuesta neuronal en la contemplación de arte (Hasson et al 2004; Kawabata and Zeki 2004) intentan probar que la subjetividad podría ser un proceso cultural sobre impuesto a la espontaneidad del cerebro ante el estímulo, una cuestión de entrenamiento y reflexión sobre lo adecuado y normal.

We speculate that shared activation during shared experiences might be one of the reasons why we can communicate-through art-impresssions and emotions that might not be possible to express through words." (Cross 2011; 7)

Si se entiende de este modo, un paradigma neuro-biológico no es una reducción o una simplificación de ninguna manera sino un añadido desde la evidencia a la compleja reflexión sobre la condición del conocimiento, o si se quiere, sobre las condiciones en las que el conocimiento es posible.

Sin embargo, una lectura crítica nos hace notar que este paradigma corre el riesgo de re-biologizar la experiencia estética, el conocimiento y la percepción. Lo cual pone en entre dicho las discusiones de la filosofía, la antropología y la psicología de todo el siglo XX que desde la lingüística general de Saussure hasta Butler, han intentado traer la cultura al centro del debate. En el caso de Canguillem y Foucault advirtiendo que siempre hay que tener mesura con nuestro propio conocimiento, dado que incluso las ciencias duras y la evidencia científica, parten de un horizonte epistémico incierto. Y un énfasis en la apreciación estética del cuerpo limita las posibilidades de la danza y corre el riesgo de capturarla como un producto de consumo donde el bailarín, en tanto que cuerpo danzante, ve vinculada su identidad a esa imagen de producto donde entra en juego lo bello, lo sexy, lo deseable.

\section{Sujeto, verdad y danza}

"Hay una cierta necesidad de separarse de lo humano que es necesaria en el proceso de rehacer lo humano"

Podemos equiparar el proceso neuronal de la danza con el que el grupo de Parma ha dado al lenguaje a través de las teorías de George Lakoff sobre la semántica corporizada. Es decir, la manera y los lugares en que el lenguaje reside biológicamente. Con las dos preguntas, suave y dura, en mente, pensemos que el lenguaje ${ }^{3}$ es al mismo tiempo más que las conexiones neuronales, y nada más que las conexiones neuronales. El lenguaje así, no sólo es abstracto, subjetivo, social, cultural, sino también es físico y sus efectos transforman físicamente (en tanto que neuronalmente) al hablante.

Entendamos entonces la danza como lenguaje de un cuerpo que comunica bailando en el espacio. La expresión de un cuerpo cuya materia blanca y cuya materia gris se reordenan a través de la disciplina física impuesta, y que constituyen al sujeto en la interacción de procesos de observación, control corporal, desplazamiento, conciencia del tiempo y el espació, la música y el silencio, comunicación con otros bailarines y conexión emocional con

3 Para una exposición en profundidad de las ideas de Lakkof y Johnson (1980) y su diferencias con otras concepciones de la lingüística (Quine, Chomsky, Jakobson) etc. 
el público. La danza implica entonces una semántica y una gramática del movimiento que permite el aprendizaje rápido, y la trasformación de datos exiguos en información compleja y acción motora (Cross et al 2011).

Nuevamente, no hace falta la evidencia de la neurobiología, aunque sin duda es eminentemente útil; se puede trabajar desde el conocimiento metafórico en terapia psicológica con supuestos similares. Así Laia Jorba Galdos (2014) reporta el uso de la danza para la integración de personalidades disociadas por medio del acercamiento de la gestualidad de las diversas personalidades.

Si la danza es una semántica que articula el movimiento dotándolo de significación y transformando el cuerpo, y si el cuerpo al transformarse incide en las posibilidades biológicas de la persona, la danza ofrece la manera de explorar la plasticidad de la identidad. Pero quizás también de encapsularla en la rigidez de la disciplina. Este es el riesgo de responder la pregunta dura desde la neurociencia, incluso con revisiones y trabajos que atenúan el carácter de las neuronas espejo, lo cierto es que la neurociencia comienza a constituirse en una episteme que permea ya la lingüística, la economía y la estética.

En la pregunta dura entran la filosofía, la antropología y la historia en el sentido que podemos establecer distintos ángulos de respuesta. La danza como lenguaje implica una historicidad y una antropología del lenguaje, sus signos, su producción y sus representaciones. La filosofía, en especial la ética, se preguntaría por las posibilidades dialógicas y transformativas de los cuerpos en movimiento ${ }^{4}$.

La antropología de la danza identifica ciertos elementos comunes en distintas tradiciones: Estética y transformación del cuerpo: (1) Tendencia a entrenar el cuerpo a grados extremos de flexibilidad. (2) Pies estirados (3) giros extremos de la cadera y las piernas. Pero es difícil, aclarar si estás son características producto de una apreciación evolutiva, de la imitación, de conceptos engranados de la masculinidad y la fertilidad o sí son signos arbitrarios. Esta dificultad nos retrotrae a la etnología, a la necesidad de escuchar a estos sujetos corpo-centricos que se debaten en el espacio social en que sus cuerpos son producidos y por lo tanto su identidad es producida. O bien, el espacio hermenéutico en el que estos sujetos interrogan a las posibilidades del conocimiento en el proceso de auto constitución.

\section{Historias de vida}

Nuestro propósito es doble, por un lado hay una preocupación desde la pedagogía de la danza sobre el papel que la disciplina tiene en la formación del bailarín. Por otro lado, una preocupación sobre los sujetos posibles en la sociedad occidental contemporánea. En concreto, en esta particular investigación, en función del cuerpo como espacio de contiendaviolencia (Turner 1989) y del cuerpo en un contexto post-materialista: expresión y calidad de vida. Pertenencia a la comunidad. "expresión y remodelación del yo".

Para eso hemos realizado cinco entrevistas semiestructuradas con cinco estudiantes universitarias o profesionistas para quienes la danza ha sido un componente importante en su vida pero que no se dedican profesionalmente a la danza, esto es así por razones de accesibilidad

4 Hay por lo tanto todo un trabajo de historia no representacional de la danza y su trasformación ver Glass, (2005), Drapatti (2004). 
pero también porque el profesional suele tener un discurso más articulado y reflexivo sobre la danza que incluye una dimensión del Cuerpo como signo y estatus dentro de unas condiciones de posibilidad (Bordieu) Un habitus que produce un sistema de esquemas de percepción y de generación de prácticas que delimitan un estilo de vida concreto. Un habitus y una clase "largas piernas, cabelleras bien cuidadas". El sentido práctico del cuerpo como activo con un valor (que en una sociedad capitalista significa precio). Las entrevistas buscaban, por una parte suscitar la reflexión y explicitar ciertas relaciones inconscientes en cuatro áreas:

- Sexualidad y género

- Dietética y Salud

- Apreciación de la danza

- Danza y entorno social

Es, todavía un trabajo preliminar que nos gustaría ampliar en un artículo con una reflexión seria de las dimensiones éticas y pedagógicas de la danza. Sin embargo, las entrevistas han mostrado elementos consistentes con los elementos teóricos que señalábamos arriba. Por cuestiones de tiempo hablaremos de las cuatro áreas un poco indistintamente mezclándolas con las entrevistas.

\subsection{Sexualidad, identidad y género}

El género aparece referenciado en las entrevistas de manera muy diversa. En tanto que identidad aparecen los roles sociales asignados. Un apunte muy interesante y que vincula el género al lugar de la danza en la sociedad lo apunta Marta, sevillana, biotecnóloga de 26 años que recuerda un episodio de su adolescencia.

En esa época Marta practicaba cerca de veinticinco horas de danza semanales. Una media de cuatro horas cada día laborable y una práctica extra los fines de semana. Refiere como en una ocasión un compañero de clase que jugaba en el Sevilla F.C. no entregó un trabajo importante y el profesorado se mostró completamente comprensivo cuando a ella, que por otro lado entregaba siempre su trabajo a tiempo, le habían recriminado no estudiar lo suficiente.

Para ella esto se debía al hecho de que la danza no es percibida como un esfuerzo:

Yo entiendo que la danza, lo que he dicho antes, que parece que estás expresando como delicadeza, pero en realidad es todo lo contrario, es tan guay esa idea porque tú en realidad lo que estás es pasando dolor, esforzándote cada día, siguiendo una disciplina. Tienes la cabeza más amueblada que un chico de tu edad que no está haciendo nada. En realidad tú estás demostrando más fortaleza en ese sentido, un rol mucho más masculino según lo que piensa la sociedad que femenino, aunque luego la gente diga “¡ay, haces ballet! Y se imagine una tía vestida de rosa de pies a cabeza. De tul y es justo lo contrario, para subir la pierna y ponerla donde la estás poniendo en realidad estás teniendo que esforzar tus abdominales al máximo, aunque tengas cara de no me importa 5 .

5 La relación de esta reflexión con la observación de los boxeadores por parte de Waqcant es directa. La disciplina es extrema aunque no esté valorada. Podría ser porque la disciplina física no se identifica con lo femenino. 
Sin embargo, se aprecia en las entrevistas cierta catalogación de las técnicas de baile que hacen que las cuatro entrevistadas señalen que prefieren ver a un bailarín que a una bailarina. Esto es consistente con las afirmaciones de la neuro-estética respecto al espacio y los movimientos amplios. Sencillamente porque el repertorio de los hombres incluye movimientos más adecuados a las características tradicionalmente reconocidas como masculinas. Esto último indica que el carácter cultural arbitrario de la danza tiene centralidad: cabe preguntarse lo que ocurriría si el repertorio de la danza incluyera esos movimientos para ambos sexos.

Dentro de la identidad juega la capacidad de comunicación y las afirmaciones que habíamos hecho sobre la danza como una semántica corporizada. En ese sentido retomamos lo que reporta una de nuestras entrevistadas. Awa, 21 años Burkina Fasso, educadora social, habla de la empatía:

(La danza) "me hacía ser más empática con los demás, entender sus movimientos. A ver, en la vida normal, lo que hacemos es hablar con los demás, pero realmente cuando bailas también estamos hablando, y cuando bailas con otra persona le estás contando una historia, y al ver bailar a otras personas podía escuchar lo que me estaban contando a mí, y las entendía.

La percepción de empatía y comunicación, que puede o no guardar relación con las resonancias neuronales, tiene sin embargo un elemento subjetivo central lo que constituye un nivel de agencia en la auto conformación y en la relación con el otro:

Tenía una compañera con la que bailé muchos años... a partir de la danza y los años nos dimos cuenta de que teníamos una relación especial. A través de la gestualidad llegué a conocer sus movimientos tan bien como conocía los míos. Éramos como una fusión. Para mi bailar es expresarse. Es hablar dejando las palabras a un lado.

Además no sólo lo refiere en el nivel comunicativo con sus compañeras sino en su comunicación con la audiencia:

Tuve una vez una actuación que hacer con una música africana de Rokia Traore, y claro a mi profesora fue quien me fue guiando los pasos y me hizo la coreografía... cada vez que hacía el baile, lloraba, y era eso, que cada vez que hacía el baile me acordaba de mi origen, de mi familia, de mi cultura. El público me lo decía, que estaban viendo la energía. Una música que estaba sintiendo, y que expresaba que echaba de menos a mi familia.

Una dimensión que subyace a la danza como acto cultural común, pero también suficientemente distinto como para que la audiencia identifique aquello que no es suyo. Que pertenece al otro cultural en el que clasifican a Awa.

Encima es que la educación social está muy cercana a lo humano, y la danza es humano, es principalmente humano... antes me hablabas de la empatía, y en la danza ayudamos a los demás y nos ayudamos a nosotros mismos. 


\subsection{Dietética y salud y auto concepto}

Las entrevistadas reportan la transformación física, en concreto modificaciones anatómicas que no se aprecian tan claramente en otros deportes. Recordemos uno de los presupuestos teóricos:

En tanto que el cuerpo se transforma no sólo en lo aparente sino en un nivel profundo (Calvo-Merino 2011) neuronal con la especialización de la materia gris y blanca el hacer se vuelve ser. La acción motora pasa a ser acción emotiva no ya en el nivel racional-reflexivo, sino en el nivel inconsciente corpóreo.

Es decir, la relación con el cuerpo que se va afirmando como contenido de la vida vivible. Esto se expresa en un doble sentido. Awa reportaba la empatía desarrollada durante el baile. Blanca, otra de nuestras entrevistadas, estudiante de medicina, muestra dos reflexiones interesantes, por un lado expresa que nunca podría dedicarse a la traumatología porque "ellos son los que te dicen que no puedes bailar". Admite el conflicto de ser consciente anatómicamente de lo que hace y entender que muchas de las modificaciones a las que ha sometido su cuerpo son lesivas y requiere suspender su juicio objetivo como futuro médico. Ella además ha dado el salto de nivel en el que la danza ha pasado a ser un interés profesional difícil de compatibilizar con la carrera.

En otro nivel, Blanca expresó de manera casual que dentro de la medicina ha tenido complicaciones personales para trabajar en la planta de Neurología porque el impacto de observar el deterioro físico irrevocable le resulta difícil de asimilar. Sin embargo no tiene problemas en trabajar en hematología porque aunque hay enfermedades mortales a una edad muy corta, no existe ese deterioro físico. Al ser preguntada si pensaba que este miedo al deterioro guardaba relación con su propio cuerpo y la centralidad de la danza, Blanca respondió:

Es una pregunta difícil, yo creo que es lo que veo en los pacientes, no tiene que ver conmigo, es decir, ver una persona de cuarenta años en silla de rueda... a lo mejor si tiene que ver con mi cuerpo. Ver que a lo mejor muere de viejo, pero que no puede mover su cuerpo, que está atrapado en una silla de rueda. A lo mejor has llegado tú antes que yo al porqué.

Blanca muestra una identificación de la normalidad física con el desplazamiento y el movimiento que le permite la danza. Intuitivamente esto es consistente con las resonancias neuronales en las que residen procesos de observación-apreciación y empatía. Y plantea una dimensión ética sobre la normalidad de los cuerpos.

Pero no sólo hacia el cuerpo sino también ante la consciencia del cuerpo:

Me impacta más una persona con una demencia, que va perdiendo la capacidad de controlar su cuerpo.

Esto es consistente con la noción de resonancia, pero también tiene un fuerte elemento cultural inconsciente. Para Blanca, la normalidad está definida dentro de una episteme 
médica. Su profesión marca claramente lo que ella ve cómo patológico y la asociación no puede deberse únicamente a un carácter neuronal aséptico respecto a las posibilidades de Blanca como sujeto virtual. La empatía no es sólo una respuesta neuronal. Implica una separación nocional entre el sujeto, el otro y las dimensiones que nos identifican: lo normal.

Dentro de esta empatía, que puede verse como una apreciación del otro y una resonancia en el uno mismo también hay un nivel transformativo al que hace referencia otra de nuestras entrevistadas. Erica tiene 22 años y es estudiante de filología, reporta que a pesar de bailar durante muchos años y disfrutar de la disciplina física, no fue hasta cambiar hacia la danza contemporánea que no experimentó comodidad con su propio cuerpo. Y reflexiona que fue en función de participar con otros cuerpos diferentes, no adecuados a las normas estrictas de la danza clásica, ni en competición etaria y estética.

Así, la coreografía en la que Erica reporta haberse sentido liberada estaba formada por ella misma, una mujer de treinta años con una condición degenerativa en los nervios de las manos y un hombre de cuarenta años y cerca de un metro noventa. Erica, quién es más bien menuda, reporta:

Valoré por primera vez el poder hacer algo bonito con mi cuerpo, que se viera bonito. Al final eso vale la pena, aunque haya algo que no sea normal. Pero éramos un cuerpo, con sus diferencias

Preguntada si la daza puede ayudar a descentrar la personalidad, Erica responde:

$\mathrm{Si}$, de hecho es muy bonito cuando sucede, cuando ves que de verdad sucede eso que rompes, es decir ya no hay género, hay una alegoría de algo. Cuando dejas de ver a la persona, es decir no dejas de ver a la persona, pero ves al bailarín. Es increíble. Sí.

La pregunta dura de la biología, como diría Heisenberg (1958), es casi metafísica, somos sólo cuerpos, pero somos algo más que cuerpos. Y apenas vislumbramos los bordes de la percepción. Una etnología es indispensable para liberar a la neuro-estética de pretensiones deterministas.

\section{Conclusiones}

Es pronto para presentar unas conclusiones definitivas, sin embargo podemos afirmar que las entrevistas en profundidad reflejan ampliamente los aspectos teóricos y trasluce la complejidad del doble paradigma: la danza como una semántica corporizada, plena de procesos inconscientes queda marcada tanto en lo afirmativo: procesos de empatía, de respuesta y solidaridad; como en lo negativo: categorías acríticas de la feminidad y la masculinidad, la condición social de la danza respecto a los hombres y la "normalidad" del cuerpo bípedo hábil, danzante, frente al cuerpo no hábil, limitado.

Del mismo modo, y dando el salto cualitativo hacia las formas críticas de toma de consciencia, observamos que la reflexión sobre estos procesos brinda espacios para la reconfiguración de la identidad y ofrecen elementos interpretativos que se pueden constituir como ese necesario deshacer lo humano que, según Butler (2010), es previo al re-hacer lo humano. 
En consecuencia, respecto al primer objetivo, el pedagógico, la reflexión tiene que partir del hecho observable de esos procesos inconscientes y su persistencia en tanto que transformaciones neuronales, es decir profundamente arraigadas en la bios del sujeto. Ahora bien, se requiere la pausa que las ciencias humanas aportan para delimitar la episteme y evitar caer en un determinismo biológico: lo que se observa, lo que la neurología comienza a observar, es imperfecto y sujeto de crítica. Las metáforas (neuronas espejo, resonancia) deben leerse con cuidado, de pronto comenzamos a comprender más claramente como las prácticas y el lenguaje persisten en la bios del sujeto, y permean duraderamente su identidad. Que deben ser tratados con más atención y que abren también posibilidades- De ahí la necesidad de explicitar la crítica. Lo cual complementa el segundo objetivo. Los espacios posibles para sujetos virtuales, que diría Foucault (2009), requieren ser explícitos sobre los valores y contenidos para garantizar al sujeto que suscitan un espacio de construcción crítico, flexible y que tome en cuenta el devenir. Espacios que permitan desde el cuerpo cuestionar lo que somos y porque lo somos.

\section{Bibliografía}

Butler, Judith. 2004. Undoing Gender. Edición: New. New York ; London: Routledge.

Bläsing, Betina, Beatriz Calvo-Merino, Emily Cross, Corinne Jola, Julianne Honisch, y Catherine Stevens. 2012. «Neurocognitive control in dance perception and performance». Acta Pshychologica 139 (2): 300-308.

Christensen, Julia F.; Calvo-Merino B. (2013). Dance as a subject for empirical aesthetics Psychology of Aesthetics, Creativity, and the Arts, Vol 7(1), Feb, 76-88.

Cross, E. S., Ticini, L. F. (2011). Neuroaesthetics and beyond: new horizons in applying the science of the brain to the art of dance. Phenomenology and the Cognitive Sciences 11(1), 5-16. (Special Issue: Dance and Cognitive Science). doi:10.1007/s11097-010-9190-y

Daprati, E., Iosa, M., Haggard, P. (2009). A dance to the Music of Time: AestheticallyRelevant Changes in Body Posture in Performing Art. Plos One, 4(3), 1-11.

Downing, P. E., Jiang, Y., Shuman, M., Kanwisher, N. (2001). A cortical area selective for visual processing of the human body. Science of Optimism and Hope, 293, 2470-2473.

Esteban, Mari Luz. 2004. Antropología del cuerpo: Géneros, itinerarios corporales, identidad y cambio. Barcelona: Bellaterra.

Foucault, Michel. 1994. Hermenéutica del sujeto. Traducido por Fernando Álvarez-Uría. Genealogía del Poder 25. Madrid: Endymion.

Foucault, Michel. 2009. El Gobierno de Sí y de los otros. Editado por Michel Senellart. Traducido por Horacio Pons. México: Fondo de Cultura Económica.

Gallese, V., Fadiga, L., Fogassi, L., \& Rizzolatti, G. (1996). Action recognition in the premotor cortex. Brain, 119, 593-609.

Heisenberg, Werner. 1958. Physics and Philosophy: The Revolution in Modern Science. New York: Harper.

Iacoboni, Marco. 2008. Mirroring People: The New Science of How We Connect with Others. New York: Farrar, Straus and Giroux.

Jang, S. H., Pollick, F. E. (2011). Experience Influences Brain Mechanisms of Watching Dance. Dance Research 29(2), 352-377. 
Jola, C., Pollick, F.E. \& Calvo-Merino, B. (2014). "Some like it hot": spectators who score high on the personality trait openness enjoy the excitement of hearing dancers breathing without music. Frontiers in Human Neuroscience, 8, 718.

Jorba Galdos, L. (2014) Creativity and dissociation. Dance/movement therapy interventions for the treatment of compartmentalized dissociation The Arts in Psychotherapy 41 467-477.

Kawabata, H, y S Zeki. (2004). «Neural correlates of Beauty». Journal of Neurophysiology 91 (4) (Abril): 1699-1705.

Lakoff, George, y Mark Johnson. 1980. Metaphors We Live by. Chicago: University of Chicago Press.

Levy, I, U Hasson, y R Malach. (2004). «One picture is worth at least a million neurons». Current Biology 14 (11): 996-1001.

Rizzolatti, G., \& Craighero, L. (2004). The mirror-neuron system. Annual Review of Neuroscience, 27, 169-192.

Sinigaglia, Corrado, y G Rizzolatti. 2011. «Through the looking glass: self and others». Consciousness and Cognition 20 (1): 64-74.

Petrini, K., Crabbe, F., Sheridan, C., \& Pollick, F. E. (2011). The Music of Your Emotions: Neural Substrates Involved in Detection of Emotional Correspondence between Auditory and Visual Music Actions. Plos One, 6(4),10. doi: e1916510.1371/journal.pone.0019165

Thiem, Annika. 2008. Unbecoming Subjects: Judith Butler, Moral Philosophy, and Critical Responsibility. New York: Fordham University Press. 